lement défaul, prendre les céniteurs parmi les Carpes de races déjà sélectionnées : Carpes cuir, Carpes miroir, Carpes à écailles. Bien qu'elles soient toutes trois de précocité égale, mes préférences vont à ces dernières.

La richesse du fond et la qualité de l'eau des étangs sont variables, comme la nature des terrains. Nous dẹons régler l'ensemencement en alevins d'après l'expérience et la connaissance que nouṣ en avons. L'épandage de chaux et d'engrais après la pèche, le faucardage d'été qui, avec les bateaux actuellement construits, est de pratique facile, permettraient une immersion d'alevins plus nombreuse ; mais n'oublions pas que, pour un étang donné, les conditions climalériques restant les mèmes, le rendement en poids est constant, quel que scit le nombre de poissons immergés. Par conséquent, pour avoir des poissons plus gros, mettons un empoissonnement moins nombreux. Dans la pratique, pour la plupart de nos étangs de Sologne, ne pas mettre plus de $\mathrm{s} \delta \mathrm{a}$ à 200 alevins de deux étés à I'hectare. Nous obtiendrons ainsi l'accroissement voulu, surtout s'il s'agit d'alevins de races sélectionnées, et nous présenterons aux amateurs le beau et bon poisson qu'ils demandent.

\title{
CAUSES ESSENTIELLES DE DÉBOIRES ET DE SUCCÉS EN CARPICULTURE
}

\author{
Par M. Michel LHÉRITIER
}

Ex-Professeur interdépartemental d'aquiculture.

L'expérience d'autrui, n'a, dit-on jamais servi à personne. J'apporte, néanmoins, ma collaboration à la rédaction d'ume publication s'adressant spécialement à des gens de métier, car je les crois plus désireux d’idées pratiques que de littérature, capables de vérifier l'exactitude de mes allégations et de profiter d'une méthode s'inspirant des lois naturelles qui m'a réussi dans les milieux les moins propices à la production, à l'éducation et la sélection des Carpes.

J'y ai aussi été incité par la conviction que, nonobstant la propagaude menée, depuis rgio, par la Compagnie des chemins de fer de Paris a Orléans, sous la direction de son distingué ingénieur des Services commerciaux, M. Potwr ; les deux Congrès de l'étang et de l'élevage de la Carpe en rgr 8 et 1924 ; les expositions piscicoles de Paris, Lyon, Limores, elc., enfin les encouragements généreusement accordés par l'Etat, l'aquiculture nationale n'a pas progressé comme l'exigeajt la situation économique.

Oui, quelques succès remarquables ont été enregistrés de ci, de là, par 
les carpiculteurs avisés ; mais, si on compare leur nombre à celui des propriétaires d'étangs de la France, force est bien de reconnaîlre que nous avons encore fort à faire pour obtenir de nos fonds d'eau la satisfaction que retirent des leurs nos collę̀ues allemands, autrichiens et polonais. Ceci après plus d'un demi-siècle d'enseignement officiel de la pisciculture (juillet 1875 ) et la création de treize Syndicats régionaux d'aquiculture et de l'Union nationale des Syndicats de l'étang.

Malgré tout ce qui a été fait, dit et écrit, n'avons-nous pas vu, la saison dernière, la majorité de nos collègues embarrassés pour la vente de leur poisson et subir la loi des revendeurs? N'entendons.nous pas les plaintes d'autres qui, ayant établi, souvent à grands frais, des frayères Dubisch, les abandonnent, wroclamant la 'aillite d'un système cependant si efficace? N'est-on pas étonné de voir présenter, dans les expositions, des reproducteurs baptisés : "Lausitz ", "Wittingau ", "Bohême ", "Franconie " ou "Aischgrund ", suivant le caprice de leurs éleveurs, mais n'ayant pas les caractères distinctifs de ces races? des alevins prétendus sélectionnés ne ressemblent pas du tout à leurs parents? un poisson catalogué Truite-Perche, lort intéressant sans doute, mais ne méritant pas cette qualification?

Pour remédier à cette situation blessante pour notre amour-propre et préjudiciable à l'économie nationale, j'exposerai les moyens paraissant les plus simples pour accroitre le rendement de fonds, assurer la production normale des alevins, l'éducation des nourrains, l'engraissement des carpes, la sélection des reproducteurs, enfin la récolte, la conservation et la vente rémunératrice des produits des étangs.

Je ne prétends pas qu'en dehors de ma méthodie il n'y a pas de succès possible ni qu'elle ne comporte aucun perfectionnement, mais j'affirme que son application donnera, dans la plupart des cas, beaucoup de satisfaction à ceux qui voudront bien la mettre en pratique, car elle s'inspire de la connaissance du milieu et des mœurs des poissons que cinquante années d'exercice de la pêche et trente de pratique de l'aquiculture m'ont permis d'acquérir mieux, sans doute, que l'étude des traités les plus complets.

La première cause des mécomptes de nos exploitants d'étangs provient de ce que l'on a, en France, l'habitude de considérer la précocité de la ponte des Carpes comme une nécessité, si bien que tous les éleveurs s'ingénient à transgresser les lois de la nature : aussi, $95 \%$ d'entre eux voientils leurs eflorts frappés de stérilité et abandonnent-ils rapidement l'emploi des frayères artificielles, cependant fort utiles à ceux qui savent les employer judicieusement.

Il ne suffit pas, en effet, de faire pondre les reproducteurs ni d'obtenir l'éclosion de leurs œufs; il faut avant it par-dessus tout assurer des vivres aux jeunes alevins qui doivent éclore; or, ces petits êtres sont telle- 
ment nombreux et doués d'un tel appétit qu'il iaut préparer les étangs d'alevinage longtemps avant de mettre les reproducteurs dans les bassins de ponte.

Il faut donc se persuader que la nourriture doit préexister dans le milieu qu'on veut peupler car cette vérité est la base de toute aquiculture rationnelle.

Si l'on considère que le "plancton " est la seule nourriture convenable pour les alevins du premier âge, la production natureile des infiniment petits qui le composent etant subordonnée aux circonstances climatériques contre lesquelles nous sommes impuissants, il apparaît clairement qu'il est absolument inutile et đu'il est mềne cruel de provoquer la naissance de milliers de petits poissons condamnés à périr de laim.

Ceux qui voudront bien y réfléchir un instant reconnaittront qu'il en va autrement, dans la nature. En effet, si I'on observe les Carpes vivant en liberté dans les étangs et les cours d'eau, on constate que ces poissons ne se reproduisent que lorsque l'enu afteint une température avoisinant $+20^{\circ}-22^{\circ}$ C., c'est-à-dire seulement à une éporque où le réchaullement de la masse liquide, amenant la décomposition des malières organiques qu'elle renferme, provoque une production considérable de petits organismes dont se nourriront les alevins à leur naissance; c'est là une leçon dont l'aquiculteur doil s'inspircr.

Le sont, sans doute, ces raisons. trop ignorées de nos jours, qui ont conduit le législateur de $18 x y$ a réserver une période d'interdiclion de pêche de deux mois, allant du 15 avril au ro juin, pour permeltre la reproduction naturelle des cyprins dans les cours d'eau de la France.

En conséquence, la mise en ouvre des reproducteurs doit donc êtro subordonnée à l'altitude de la région où l'on opère, à la nature des eaux, à l'exposition du milieu à exploiter et à lambiance.

Je ne saurais donc trop insister auprès de mes collègues carpiculteurs pour qu'ils s'assurent, par l'examen préalable de leurs eaux, que leurs alevins trouveront à manger dès leur naissance dans les étangs qui leur sont réservés.

Une autre erreur consiste à confondre la frayère artificielle avec l'étang d'alevinage. La première doit être exclusivement la chambre nuptiale où mâles et femelles prendront leurs ébats amoureux dans les conditions les plus favorables à la ponte ct à la fécondation des œufs, puis à leur éclosion, tandis que l'étang, - ou mieux, les étangs d'alevinage - sont des pièces d'eau desquelles on auma écarté le mieux possible les causes naturelles de destruction des alevins et où ceux-ci trouveront, durant leur premier été, une nourriture fort abondante et appropriée à leurs besoins.

Les succès constants de la technique allemande sont dûs à la stricte observation de ces vérités.

Donc, avant toute tentative de production d'alevins, l'exploitant doit 
s'inspirer des conditions particulières du milieu où il vcut opérer et baser ses travaux sur l'époque habituelle de la reproduction naturelle des poissons dans ses parages. Ensuite, par des procédés appropriés à ce milieu, il pourra accroître la production du zooplancton et souvent même avancer avantageusement l'époque de la ponte, car il est certain que, dans ces conditions, plus tôt les alevins naîtront, plus ils grandiront avant l'hiver.

Mais, comme les jours, les années se suivent et ne se ressemblent pas ; j'ai vu, dans le même milieu, les Carpes frayer une fois le r 5 mai et une autre fois Je r/f juillet, alors que l'époque habituelle de la ponte était, 9 fors sur to, comprise entre le 22 et le 26 juin: Les alevins de mai restèrent peu nombreux, mais atteignirent une taille exceptionnelle ; ceux de juillet, en très grand nombre, restèrent de très petite taille, alors que ceux naissant habituellement en juin atteignent généralement un poids moyen de 20 grammes pièce à la fin de leur premier été.

Une troisième erreur est d'oublier que le développement de tous les êtres est, avant tout, une question de nourriture, et de croire qu'il suffit aux poissons d'être dans i'eau pour être heureux, ainsi que le prétend un dicton populaire. Or, il n'en est rien car, comme les cochons, les carpes ne s'engraissent pas avec de l'eau claire ; leur élevage ne peut être lucratif que si elles ont constamment à leur disposition la ration maxima qu'elles pourront absorber ; c'est là un principe de zootechnie qui s'applique à l'élevage des poissons comme à celui de tous les autres animaux : bœufs, porcs, moutons, lapins, volailles.

Un animal qui recevrait seulement la ration d'entretien, absorberait pendant sa vie une masse énorme de nourriture et ce serait une ruine. Il n'y a que la nourriture donnée en sus de ce qu'il faut pour entretenir la vie qui produit de la viande, du lait et du travail ; plus cette nourriture supplémentaire est abondante plus le profit est grand.

Tout cela est admis sans conteste par tous Jes éleveurs de bestiaux et doit l'être, également, par tous les éleveurs de poissons, car c'est là, la cause essentielle du succès de la méthode Dubisch. Après avoir fait naître les alevins, elle les conduit successivement dans des petits étangs établis dans des conditions spéciales et traités convenablement pour assurer le fourmillement du zooplancton dont ils sont avides et assure ainsi leur développement rapide. Il en est de mème pour les poissons du deuxième et du troisième âge, ce qui, dans un laps de temps très court, donne le maximum de rendement. 